\title{
Artin characters, Hurwitz trees and the lifting problem
}

\author{
Louis Brewis and Stefan Wewers
}

\begin{abstract}
We study finite groups of automorphisms of the $p$-adic open disk. In particular, we generalize results of Green, Matignon and Henrio from cyclic groups of order $p$ to arbitrary finite groups. As an application, we produce a counterexample to a question of Chinburg, Guralnick and Harbater, concerning the local lifting problem for generalized quaternion groups.
\end{abstract}

\section{Introduction}

1.1 Automorphisms of the $p$-adic disk Let $K$ be a nonarchimedian local field of characteristic zero, with residue field of characteristic $p$. Let $\mathrm{Y}=(\operatorname{Spf} R[[z]]) \otimes K$ be the rigid open unit disk over $K$. In this note we are concerned with the following question:

How can a finite group $G$ act on $Y$ ?

Let $G \subset \operatorname{Aut}_{K}(\mathrm{Y})$ be a finite group of automorphisms of $\mathrm{Y}$; alternatively, we may regard $G$ as a subgroup of $\operatorname{Aut}_{R}(R[[z]])$. It is not hard to show that $G=P \rtimes C$ is the semi-direct product of a $p$-group $P$ and a cyclic group $C$ of order prime to $p$. Moreover, after a suitable change of parameter the action of a generator $\sigma$ of $C$ is of the form

$$
\sigma(z)=\zeta \cdot z
$$

where $\zeta \in K^{\times}$is a root of unity. In particular, the case where $G$ is of order prime to $p$ is well understood.

In their fundamental paper [7], Green and Matignon studied the case of a cyclic group $G$ of order $p$ - the first interesting case. Their results establish a precise relation between

- the 'geometry of fixed points', i.e. the relative position inside $Y$ of the fixed points of a generator of $G$, and

- the ramification of the Galois cover

$$
\mathrm{Y} \longrightarrow \mathrm{X}:=\mathrm{Y} / G
$$

with respect to the Gauss norm of an arbitrary subdisk of $Y$. 
These results were sharpened by Henrio ([9], [8]), in two ways. Firstly, he introduced the notion of Hurwitz tree, an essentially combinatorial object which encodes both the geometry of fixed points of an automorphism of order $p$ and the associated ramification data. Secondly, he proved that every Hurwitz tree can be realized by an actual automorphism of $\mathrm{Y}$.

In the present paper we generalize the notion of Hurwitz tree to the case of an arbitrary group of automorphisms of Y. Following [8], we define a Hurwitz tree as an oriented tree equipped with a metric and some further additional data, satisfying certain conditions. Our main results associates to a group of automorphisms $G$ of the disk a Hurwitz tree $\mathcal{T}$. By construction, the metric tree underlying $\mathcal{T}$ describes the geometry of fixed points of the $G$-action, and the additional data reflect the ramification of the $G$-action with respect to certain valuations of $R[[z]]$. In the special case considered in [8], these additional data consist of certain numbers attached to each vertex and edge of the tree. Our generalization is based on replacing these numbers by virtual characters of $G$, which are constructed using Huber's ramification theory [10].

In this paper we do not consider the problem of realizing a given Hurwitz tree by a $G$-action of the disk (which is solved in [8] for $G \cong \mathbb{Z} / p$ and in [3] for $G \cong \mathbb{Z} / p \rtimes \mathbb{Z} / m$, with $(m, p)=1)$. This explains why our Hurwitz trees are not equipped with differential data, as in [8] and [3]. For a general theory of Hurwitz trees with diffential data, see the first author's upcoming thesis [4].

1.2 New obstructions for the local lifting problem Let $k$ be an algebraically closed field of positive characteristic $p$. Let $G$ be a finite group. A local $G$-action is a faithful $k$-linear action $\phi: G \hookrightarrow \operatorname{Aut}_{k}(k[[z]])$ of $G$ on a ring of power series over $k$ in one variable. The local lifting problem for $\phi$ concerns the following question:

Does $\phi$ lift to an action $\phi_{R}: G \hookrightarrow R[[z]]$, where $R$ is a discrete valuation ring of characteristic 0 with residue field $k$ ?

If it does, we say that $\phi$ lifts to characteristic 0 , or that $\phi$ is liftable.

In [2], Bertin has formulated a necessary condition for liftability of $\phi$, which is commonly called the Bertin obstruction. If this necessary condition holds then we say that the Bertin obstruction for $\phi$ vanishes. From our main result we directly obtain a new necessary condition for liftability of local $G$-actions, which is a refinement of the Bertin obstruction.

We give examples of local $G$-actions (the so-called simple quaternion actions) with vanishing Bertin obstruction, but for which our new condition does not hold - and which therefore do not lift to characteristic zero. Here the group $G$ is a generalized quaternion group $Q_{2^{n+1}}$ of order $2^{n+1}$, with $n \geq 2$, and $k$ has characteristic 2.

Chinburg, Guralnick and Harbater ([6], [5]) call a group $G$ a local Bertin group if the Bertin obstruction of every local $G$-action vanishes. They call $G$ a local Oort group if every local $G$-action lifts to characteristic zero. They prove that the generalized quaternion groups $Q_{2^{n+1}}$ are local Bertin groups for $n \geq 3$. 
However, our result shows that these groups are not local Oort groups. This answers Question 1.3 of [5] negatively.

\section{Hurwitz trees}

A Hurwitz tree $\mathcal{T}$ consists of an oriented metric tree $T$ and certain additional data attached to each vertex and edge of $T$, satisfying certain conditions. These additional data are related to a finite group $G$ and a prime number $p$. Precise definitions will be given in the following Sections 2.1, 2.2 and 2.3. We postpone all motivation and explanation of these definitions to Section 3.

In Section 2.4 we discuss the notion of density. Later on in Section 4 this will be our main tool for showing that certain Hurwitz trees and, therefore, certain group actions on the disk, are impossible.

2.1 Let $G$ be a finite group. We denote by $R(G)$ the Grothendieck group of the category of $\mathbb{C}[G]$-modules of finite type $([13], \S 14.1)$. We may identify elements of $R(G)$ with their virtual characters $\chi: G \rightarrow \mathbb{C}$. We denote by $R^{+}(G) \subset R(G)$ the submonoid of true characters.

We write $1_{G} \in R^{+}(G)$ for the unit character, $r_{G} \in R^{+}(G)$ for the regular character and $u_{G}=r_{G}-1_{G} \in R^{+}(G)$ for the augmentation character.

Given two characters $\chi_{1}, \chi_{2} \in R(G)$, their scalar product is defined as

$$
\left\langle\chi_{1}, \chi_{2}\right\rangle_{G}:=|G|^{-1} \cdot \sum_{\sigma \in G} \overline{\chi_{1}(\sigma)} \chi_{2}(\sigma) .
$$

In the following, we will mostly identify an element of $R(G)$ with the induced linear form on $R(G)$, i.e. we consider $\chi \in R(G)$ as a map

$$
R(G) \rightarrow \mathbb{Z}, \quad \psi \mapsto \chi(\psi):=\langle\psi, \chi\rangle_{G} .
$$

Given a group homomorphism $\phi: H \rightarrow G$, we obtain $\mathbb{Z}$-linear maps (restriction and induction)

$$
\phi^{*}: R(G) \rightarrow R(H), \quad \phi_{*}: R(H) \rightarrow R(G) .
$$

They are related by the Frobenius reciprocity formula:

$$
\left\langle\psi, \phi_{*}(\chi)\right\rangle_{G}=\left\langle\phi^{*}(\psi), \chi\right\rangle_{H} .
$$

If $H$ is a subgroup of $G$ and $\phi$ the canonical injection, then we will simply write $\left.\psi\right|_{H}$ instead of $\phi^{*}(\psi)$ and $\chi^{*}$ instead of $\phi_{*} \chi$. See e.g. [14], VI, $\S 1$.

A $\mathbb{Q}$-valued virtual character is an element of the group $R(G, \mathbb{Q}):=R(G) \otimes_{\mathbb{Z}}$ $\mathbb{Q}$. We will consider an element $\chi \in R(G, \mathbb{Q})$ either as a class function $\chi: G \rightarrow \mathbb{C}$ or as a $\mathbb{Z}$-linear map $\chi: R(G) \rightarrow \mathbb{Q}$. The submonoid $R^{+}(G, \mathbb{Q}) \subset R(G, \mathbb{Q})$ consists, by definition, of the elements $\chi \in R(G, \mathbb{Q})$ with $\chi(\psi) \geq 0$ for all $\psi \in R^{+}(G)$. An important example of such a $\mathbb{Q}$-valued character is the following: 
Definition 2.1 Let $p$ be a prime number and $G=\langle\sigma\rangle \cong \mathbb{Z} / p^{m} \mathbb{Z}$ be a finite cyclic group of order $p^{m}$, with $m \geq 0$. We define an element $\delta_{G}^{\text {mult }} \in R(G, \mathbb{Q})$ via the following class function. For $a \not \equiv 0\left(\bmod p^{m}\right)$ we set

$$
\delta_{G}^{\mathrm{mult}}\left(\sigma^{a}\right):=-\frac{p^{i+1}}{p-1},
$$

where $i:=\operatorname{ord}_{p}(a)<m$ is the exponent of $p$ in $a$; furthermore,

$$
\delta_{G}^{\text {mult }}(1):=-\sum_{a=1}^{p^{m}-1} \delta_{G}^{\text {mult }}\left(\sigma^{a}\right)=m p^{m} .
$$

Let $\chi \in R^{+}(G)$ be an irreducible character of $G$ of order $p^{n}$ (with $0 \leq n \leq$ $m)$. One easily checks that

$$
\delta_{G}^{\text {mult }}(\chi)= \begin{cases}\frac{n p-n+1}{p-1}, & n>0, \\ 0, & \chi=1_{G} .\end{cases}
$$

It follows that $\delta_{G}^{\text {mult }} \in R^{+}(G, \mathbb{Q})$.

(The superscript mult ${ }^{\text {stands for multiplicative and was chosen because } \delta^{\text {mult }}}$ describes the ramification of a torsor under the multiplicative group scheme $\mu_{p^{n}}$. See Lemma 3.8.)

\section{$2.2 \quad$ Metric trees}

Definition 2.2 Let $T$ be a connected tree, with set of vertices $V$ and set of edges $E$ and with one distinguished vertex $v_{0} \in V$, called the root. We call $T$ a rooted tree if the root $v_{0}$ is connected to a unique edge $e_{0} \in E$ (which we call the trunk of $T$ ).

A rooted tree $T$ carries a natural orientation, determined by source and target maps $s, t: E \rightarrow V$, as follows. Given an edge $e \in E$, the source $s(e)$ (resp. the target $t(e))$ is the vertex adjacent to $e$ contained in same connected component of $T \backslash\{e\}$ as $v_{0}$ (resp. in the connected component not containing $\left.v_{0}\right)$. If $v=s(e)$ and $v^{\prime}=t(e)$ we call $v^{\prime}$ a successor of $v$; notation: $v \rightarrow v^{\prime}$. There is a natural partial ordering $\leq$ on $V$, where $v_{1} \leq v_{2}$ if and only if there is an oriented path starting from $v_{1}$ and ending at $v_{2}$.

It is clear that the root $v_{0}$ is the unique minimal vertex with respect to this ordering. A maximal vertex is called a leaf. We write $B \subset V$ for the set of all leaves. It follows from Definition 2.2 that $B$ is nonempty and does not contain the root $v_{0}$. For any vertex $v$ we define

$$
B_{v}:=\{b \in B \mid v \leq b\}
$$

as the set of leaves which can be reached from $v$ along an oriented path.

Definition 2.3 Let $T$ be a rooted tree. A metric on $T$ is given by a map $\epsilon: E \rightarrow \mathbb{Q}_{\geq 0}, e \mapsto \epsilon_{e}$ such that $\epsilon_{e}=0$ if and only if $t(e)$ is a leaf. We call $\epsilon_{e}$ the thickness of the edge $e$. The pair $(T, \epsilon)$ is called a metric tree. Sometimes we write $T$ instead of $(T, \epsilon)$, if no confusion can arise. 
2.3 Hurwitz trees Let $G$ be a finite group and $p$ a prime number.

Definition 2.4 A Hurwitz tree of type $(G, p)$ is a datum $\mathcal{T}=\left(T,\left[G_{v}\right], a_{e}, \delta_{v}\right)$, where

- $T=(T, \epsilon)$ is a metric tree (with root $v_{0}$, trunk $e_{0}$ and set of leaves $B$ ),

- $\left[G_{v}\right]$ is the conjugacy class of a subgroup $G_{v} \subset G$, for every vertex $v$ of $T$,

- $a_{e} \in R^{+}(G)$ is a character of $G$, for every edge $e$ of $T$,

- $\delta_{v} \in R^{+}(G, \mathbb{Q})$ is a $\mathbb{Q}$-valued character of $G$, for all vertices $v$.

We call $G_{v}$ the monodromy group and $\delta_{v}$ the depth of the vertex $v$. We call $a_{e}$ the Artin character of the edge $e \in E$.

The datum $\mathcal{T}$ is required to satisfy the following conditions:

(H1) Let $v$ be a vertex. Then, up to conjugation in $G$, we have

$$
G_{v^{\prime}} \subset G_{v}
$$

for every successor $v^{\prime}$ of $v$. Moreover, we have

$$
\sum_{v \rightarrow v^{\prime}}\left[G_{v}: G_{v^{\prime}}\right]>1
$$

except if $v=v_{0}$ is the root, in which case there exists exactly one successor $v^{\prime}$ and we have $G_{v}=G_{v^{\prime}}=G$.

(H2) The group $G_{b}$ is nontrivial and cyclic, for every leaf $b \in B$.

(H3) For all $e \in E$ we have

$$
a_{e}= \begin{cases}\sum_{t(e)=s\left(e^{\prime}\right)} a_{e^{\prime}}, & t(e) \notin B, \\ u_{G_{b}}^{*}, & b=t(e) \in B .\end{cases}
$$

(H4) For all $e \in E$ we have

$$
\delta_{t(e)}=\delta_{s(e)}+\epsilon_{e} \cdot s_{e},
$$

where $s_{e}:=a_{e}-u_{G_{t(e)}}^{*} \in R(G)$.

(H5) For $b \in B$ we let $P_{b} \subset G_{b}$ denote the Sylow $p$-subgroup of $G_{b}$. Then

$$
\delta_{b}=\left(\delta_{P_{b}}^{\text {mult }}\right)^{*} .
$$

Here $\delta_{P_{b}}^{\text {mult }}$ is given by Definition 2.1 (and depends on the prime $p$ ).

We set

$$
\delta_{\mathcal{T}}:=\delta_{v_{0}}, \quad a_{\mathcal{T}}:=a_{e_{0}},
$$

which we call the depth and the Artin character of the Hurwitz tree $\mathcal{T}$. 
Remark 2.5 Let $\mathcal{T}=\left(T,\left[G_{v}\right], a_{e}, \delta_{v}\right)$ be a Hurwitz tree, as in Definition 2.4.

(i) Using Condition (H1), it is easy to show that there exists a metric tree $\tilde{T}$, together with an action of $G$ which fixes the root, such that

- $T=\tilde{T} / G$,

- for every vertex $v$ of $T$ there exists a vertex $\tilde{v}$ of $\tilde{T}$ above $v$ such that $G_{v}$ is the stabilizer of $\tilde{v}$.

In fact, a metric tree $\tilde{T}$ with $G$-action as above corresponds one-to-one to the datum $\left(T, G_{v}\right)$, satifying condition (H1).

(ii) Condition (H3) is equivalent to the following claim: for all edges $e$ we have

$$
a_{e}=\sum_{b \in B_{t(e)}} u_{G_{b}}^{*} .
$$

This follows immediately from induction over the tree $T$.

(iii) It follows from (ii) that the Artin characters $a_{e}$ are already determined by the tree $T$ and the conjugacy classes of (cyclic) subgroups $\left(\left[G_{b}\right]\right)_{b \in B}$. Moreover, using (H4) and (H5) we see that the depth $\delta_{v}$ is determined by the metrized tree $(T, \epsilon)$ and the conjugacy classes $\left(\left[G_{v}\right]\right)_{v \in V}$.

(iv) By (iii) the Hurwitz tree $\mathcal{T}$ is uniquely determined by the datum $\left(T,\left[G_{v}\right]\right)$ which corresponds, by (i), to a metric tree $\tilde{T}$ with a $G$-action. However, it is a highly nontrivial condition, for a metric tree $\tilde{T}$ with $G$-action, to come from a Hurwitz tree $\mathcal{T}$ of type $(G, p)$.

2.4 Densities We fix a Hurwitz tree $\mathcal{T}=\left(T,\left[G_{v}\right], a_{e}, \delta_{v}\right)$ of type $(G, p)$, with set of leaves $B$.

Definition 2.6 (i) Let $b_{1}, b_{2} \in B$ be two distinct leaves. The inverse distance of $b_{1}$ and $b_{2}$ is the positive rational number $d\left(b_{1}, b_{2}\right) \in \mathbb{Q}_{>0}$ defined as follows. Let $\left(v_{0}, v_{1}, \ldots, v_{r}\right)$ be the longest oriented path in $T$ starting from the root $v_{0}$ and ending in a vertex $v_{r} \notin B$ with $v_{r} \leq b_{1}, b_{2}$. For $i=1, \ldots, r$ let $e_{i}$ be the edge with $s\left(e_{i}\right)=v_{i-1}$ and $t\left(e_{i}\right)=v_{i}$. Then we set

$$
d\left(b_{1}, b_{2}\right):=\sum_{i=1}^{r} \epsilon_{e_{i}} .
$$

(ii) Let $A \subset B$ be a nonempty set of leaves and $b \in A$. The density of $A$ at $b$ is the rational number

$$
d(A, b):=\sum_{b^{\prime} \in A \backslash\{b\}} d\left(b, b^{\prime}\right) .
$$

Note that $d(A, b)$ only depends on $A, b$ and the metrized tree $T$. 
Lemma 2.7 Let $A, b$ be as in Definition 2.6.

(i) Let $\left(v_{0}, v_{1}, \ldots, v_{r}, b\right)$ be the unique oriented path from the root to $b$. For $i=1, \ldots, r$ let $e_{i}$ be the edge with $s\left(e_{i}\right)=v_{i-1}$ and $t\left(e_{i}\right)=v_{i}$. Then

$$
d(A, b)=\sum_{i=1}^{r} \epsilon_{e_{i}} \cdot n\left(A, v_{i}\right),
$$

where

$$
n(A, v):=\left|\left\{b^{\prime} \in A \mid b^{\prime} \neq b, v \leq b^{\prime}\right\}\right| .
$$

(ii) Let $\chi \in R(G)^{+}$be a character such that

$$
\left\langle\chi, u_{G_{a}}^{*}\right\rangle_{G}= \begin{cases}m, & a \in A, \\ 0, & a \in B \backslash A,\end{cases}
$$

where $m:=\left\langle\chi, u_{G}\right\rangle_{G}$. Then

$$
m \cdot d(A, b)=\delta_{b}(\chi)-\delta_{v_{0}}(\chi) .
$$

Proof: The proof of (i) follows from a simple induction argument which we leave to the reader. For the proof of (ii) we may assume that $G_{b} \subset G_{v_{r}} \subset$ $G_{v_{r-1}} \subset \ldots \subset G$, by Condition (H1) of Definition 2.4. We deduce the following sequence of inequalities

$$
m=\left\langle\chi, u_{G_{b}}^{*}\right\rangle \leq\left\langle\chi, u_{G_{v_{r}}}^{*}\right\rangle \leq \ldots \leq\left\langle\chi, u_{G}\right\rangle=m,
$$

which, a posteriori, turn out to be equalities. Using Remark 2.5 and the hypothesis on $\chi$ we therefore get

$$
s_{e_{i}}(\chi)=a_{e_{i}}(\chi)-m=\sum_{a \in B_{v_{i}}}\left\langle\chi, u_{G_{a}}^{*}\right\rangle-m=m \cdot n\left(A, v_{i}\right) .
$$

Now we compute:

$$
\begin{aligned}
\delta_{b}(\chi)-\delta_{v_{0}}(\chi) & =\sum_{i=1}^{r} \delta_{v_{i}}(\chi)-\delta_{v_{i-1}}(\chi) \\
& \stackrel{(\mathrm{H} 3)}{=} \quad \sum_{i=1}^{r} \epsilon_{e_{i}} \cdot s_{e_{i}}(\chi) \\
& \stackrel{(2)}{=} \quad m \cdot \sum_{i} \epsilon_{e_{i}} \cdot n\left(A, v_{i}\right) \\
& \stackrel{(\mathrm{i})}{=} \quad m \cdot d(A, b) .
\end{aligned}
$$


Example 2.8 Assume that $G=\mathbb{Z} / p^{n} \mathbb{Z}$ and let $b \in B$ such that $G_{b}=G$. Let $\chi$ be an irreducible character of $G$ with trivial kernel. Then by Lemma 2.7, (H5) and (1) we have

$$
d(B, b)=\frac{n p-n+1}{p-1}-\delta_{\mathcal{T}}(\chi)
$$

If $\delta_{\mathcal{T}}=0$ (which is the interesting case for us) we thus get a simple formula for the density $d(B, b)$ which puts a strong restriction on the metric of the tree $T$.

\section{Group actions on the disk}

3.1 We fix the following notation. Let $K$ be a field of characteristic zero which is complete with respect to a discrete (additive) valuation val $: K^{\times} \rightarrow \mathbb{Q}$. Let $R \subset K$ denote the valuation ring and $\pi$ a prime element of $R$. We assume that the residue field $k=R /(\pi)$ is algebraically closed of characteristic $p>0$. We also assume that $\operatorname{val}(p)=1$.

We fix an open rigid-analytic disk $\mathrm{Y}$ over $K$ and a subgroup $G \subset \operatorname{Aut}_{K}(\mathrm{Y})$. We assume that there exists at least one fixed point, i.e. a point in $Y$ with a nontrivial stabilizer. The goal of this section is to attach to $(\mathrm{Y}, G)$ a Hurwitz tree $\mathcal{T}=\left(T,\left[G_{v}\right], a_{e}, \delta_{v}\right)$ of type $(G, p)$.

This construction is based on Huber's theory of Artin and Swan characters for rigid-analytic curves ([10]). But since we only consider a very special case (a disk), we can do everything in an elementary and self-contained way, and we do not have to actually use any of the results of [10].

3.2 The depth character At the beginning we shall work with a slightly more general situation than announced above. The ring $A$ will either denote the ring of formal power series $R[[z]]$ or the ring $R\{z\}$ of convergent powers series in $z$. It gives rise to a formal scheme $\mathcal{Y}:=\operatorname{Spf} A$ and a rigid-analytic space $\mathrm{Y}:=\mathcal{Y} \otimes K$. In the first case, $\mathrm{Y}$ is an open disk, i.e.

$$
\mathrm{Y}=\{z \mid \operatorname{val}(z)>0\} .
$$

In the second case it is a closed disk, and we have a bijection

$$
\mathrm{Y}=\{z \mid \operatorname{val}(z) \geq 0\} .
$$

We let valY $: A \backslash\{0\} \rightarrow \mathbb{Q}$ denote the Gauss valuation, i.e.

$$
\operatorname{val}_{Y}\left(\sum a_{i} z^{i}\right)=\min _{i} \operatorname{val}\left(a_{i}\right)
$$

We set $\bar{A}:=A /(\pi)$ and let $\bar{f} \in \bar{A}$ denote the image of $f \in A$. We have $\bar{A}=k[[z]]$ or $\bar{A}=k[z]$.

Suppose we are given a finite subgroup $G \subset \operatorname{Aut}_{K}(\mathrm{Y})$ of automorphisms of $\mathrm{Y}$. The action of $G$ extends uniquely to the formal model $\mathcal{Y}$ and hence induces an action of $G$ on the ring $A$. 
Our first goal is to define an invariant $\delta_{Y}^{G} \in R^{+}(G, \mathbb{Q})$, called the depth character. It measures the ramification of $G$ with respect to valy, i.e. the amount to which the induced map $G \rightarrow \operatorname{Aut}_{k}(\bar{A})$ fails to be injective.

Let $I \triangleleft G$ be the inertia group with respect to valY, i.e. the normal subgroup consisting of elements $\sigma \in G$ with $\operatorname{val}_{Y}(\sigma(z)-z)>0$.

Definition 3.1 The depth character associated to $(\mathrm{Y}, G)$ is the $\mathbb{Q}$-valued character $\delta_{\mathrm{Y}}^{G} \in R(G, \mathbb{Q})$ associated to the following class function:

$$
\delta_{Y}^{G}(\sigma):=-|G| \cdot \operatorname{val}_{Y}(\sigma(z)-z)
$$

for $\sigma \in G \backslash\{1\}$ and

$$
\delta_{Y}^{G}(1):=-\sum_{\sigma \neq 1} \delta_{Y}^{G}(\sigma) .
$$

By definition we have $\delta_{\mathrm{Y}}^{G}=0$ if and only if $I=\{1\}$.

A priori, it is not clear why $\delta_{\mathrm{Y}}^{G}$ is a class function, as it seems to depend on the choice of the parameter $z$. However, the following lemma shows that it actually does not.

Lemma 3.2 Fix an element $\sigma \in I \backslash\{1\}$. Then:

(i) For all $f \in A$ we have

$$
\operatorname{val}_{Y}(\sigma(f)-f) \geq \operatorname{val}_{Y}(\sigma(z)-z) .
$$

(ii) We have equality in (i) if and only if $\mathrm{d} \bar{f} \neq 0$.

Therefore, $\delta_{\mathrm{Y}}^{G}(\sigma)$ is independent of the choice of the parameter $z$ and only depends on the conjugacy class of $\sigma$ in $G$.

Proof: Since $\sigma \in I \backslash\{1\}$, there exists an element $a \in R$ such that

$$
\operatorname{val}(a)=\min \left\{\operatorname{val}_{Y}(\sigma(f)-f) \mid f \in A\right\}>0 .
$$

For $g \in A$ with $\operatorname{valy}(g) \geq \operatorname{val}(a)$ we define

$$
[g]:=\overline{(g / a)} \in \bar{A} .
$$

We claim that the map

$$
\partial: \bar{A} \rightarrow \bar{A}, \quad \bar{f} \mapsto[\sigma(f)-f] .
$$

is a derivation. Indeed, it is clearly $k$-linear, and for $f, g \in A$ we get:

$$
\begin{aligned}
\partial(\overline{f g}) & =[\sigma(f)(\sigma(g)-g)+g(\sigma(f)-f)] \\
& =\bar{f} \partial(\bar{g})+\bar{g} \partial(\bar{f})+[(\sigma(f)-f)(\sigma(g)-g)] \\
& =\bar{f} \partial(\bar{g})+\bar{g} \partial(\bar{f}) .
\end{aligned}
$$

This proves the claim. But $\partial \neq 0$ by definition. It follows that $\partial(\bar{f})=0$ if and only if $\mathrm{d} \bar{f}=0$. This proves the lemma. 
Lemma 3.3 We have $\delta_{Y}^{G} \in R^{+}(G, \mathbb{Q})$.

Proof: One easily checks (using Lemma 3.2) that for $h \in \mathbb{Q}_{\geq 0}$ the set

$$
G_{h}:=\left\{\sigma \in G \mid \operatorname{val}_{Y}(\sigma(z)-z) \geq h\right\}
$$

is a normal subgroup of $G$. Let

$$
0<h_{1}<\ldots<h_{m}
$$

be the breaks, i.e. the positive values of the function

$$
G \backslash\{1\} \rightarrow \mathbb{Q}, \quad \sigma \mapsto \operatorname{val}_{Y}(\sigma(z)-z) .
$$

One verifies, by a direct computation, that

$$
\delta_{\mathrm{Y}}^{G}=\sum_{i=1}^{m} \lambda_{i} \cdot u_{G_{h_{i}}}^{*},
$$

where

$$
\lambda_{i}:=\left|G_{h_{i}}\right|\left(h_{i}-h_{i-1}\right)>0 .
$$

and $h_{0}:=0$. The lemma follows from the positivity of $\lambda_{i}$.

3.3 The Artin character We continue with the notation introduced above. But from now on we assume that $A=R[[z]]$, i.e. that $\mathrm{Y}$ is an open disk. Our goal is to define an Artin character $a_{Y}^{G} \in R^{+}(G)$ which describes the action of $G$ on the boundary of $\mathrm{Y}$.

We define

$$
\#_{\curlyvee} f:=\operatorname{ord}_{z}\left(\overline{f / p^{\text {val }_{Y}(f)}}\right) .
$$

Here $\operatorname{ord}_{z}: k[[z]] \rightarrow \mathbb{Z} \cup\{\infty\}$ is the usual order function and $p^{\operatorname{val}_{\curlyvee}(f)} \in R$ is an arbitrary element with valuation $\operatorname{val}_{Y}(f)$. The Weierstrass preparation theorem shows that $\#_{\mathrm{Y}} f$ is the number of zeroes of $f$ on $\mathrm{Y}$, counted with multiplicity.

Definition 3.4 The Artin character of $(\mathrm{Y}, G)$ is the element of $R^{+}(G)$ associated to the class function defined by

$$
a_{Y}^{G}(\sigma):=-\#_{\Upsilon}(\sigma(z)-z), \quad \text { for } \sigma \neq 1
$$

and

$$
a_{Y}^{G}(1):=-\sum_{\sigma \neq 1} a_{Y}^{G}(\sigma)
$$

To see that $a_{Y}^{G}$ is indeed a character we relate it to the permutation representation arising from the set of fixed points. For $\sigma \in G \backslash\{1\}$ let $\Delta_{\sigma} \subset \mathrm{Y}(\bar{K})$ denote the set of (geometric) fixed points of $\sigma$. Set

$$
\Delta:=\cup_{\sigma \neq 1} \Delta_{\sigma} .
$$

This is a finite $G$-set. Let $B:=\Delta / G$ denote the orbit space. Choose, for each $b \in B$, an element $y \in \Delta$ belonging to $b$ and let $G_{b} \subset G$ denote the stabilizer of $y$. 
Proposition 3.5 We have

$$
a_{\curlyvee}^{G}=\sum_{b \in B} u_{G_{b}}^{*} .
$$

In particular, $a_{Y}^{G}$ is an element of $R^{+}(G)$.

Proof: Fix an element $\sigma \in G \backslash\{1\}$. Then $\Delta_{\sigma}$ is the set of zeroes of the function $f_{\sigma}:=\sigma(z)-z$. An easy local calculation, coupled with the assumption that $\sigma$ has finite order and that $\operatorname{char}(K)=0$, shows that all zeroes of $f_{\sigma}$ are simple (cf. [7], §II.1). Therefore, by Definition 3.4 and the Weierstrass preparation theorem we have

$$
a_{\Upsilon}^{G}(\sigma)=-\# \mathrm{Y} f_{\sigma}=-\left|\Delta_{\sigma}\right| .
$$

The proposition follows immediately.

The next proposition is the key result behind the construction of the Hurwitz tree associated to $(\mathrm{Y}, G)$.

Proposition 3.6 Let $\mathrm{D} \subset \mathrm{Y}$ be a closed disk which contains the set $\Delta$ and is fixed by the action of $G$. Let $\mathrm{E} \subset \mathrm{D}$ denote the residue class of a $K$-rational point $y$ in D. Let $H \subset G$ denote the stabilizer of $\mathrm{E}$. Then

$$
\delta_{\mathrm{D}}^{G}=\left(\delta_{\mathrm{E}}^{H}\right)^{*}=\delta_{\mathrm{Y}}^{G}+|G| \cdot \epsilon \cdot s_{\mathrm{Y}}^{G},
$$

where $s_{Y}^{G}:=a_{\curlyvee}^{G}-u_{G}$ and where $\epsilon \in \mathbb{Q}_{>0}$ is the thickness of the annulus $Y \backslash \mathrm{D}$.

Proof: After a change of parameter we may assume that the point $y$ is given by the equation $z=0$. Then

$$
\mathrm{D}=\{z \mid \operatorname{val}(z) \geq \epsilon\}, \quad \mathrm{E}=\{z \mid \operatorname{val}(z)>\epsilon\} .
$$

After replacing $K$ by some finite extension, we may further assume that there exists an element $a \in R$ with $\operatorname{val}(a)=\epsilon$. We obtain formal models $\mathrm{D}=$ $(\operatorname{Spf} R\{w\}) \otimes_{R} K$ and $\mathrm{E}=(\operatorname{Spf} R[[w]]) \otimes K$, where $w:=a^{-1} z$. By definition, we have $\operatorname{val}_{D}=\left.\operatorname{val}_{E}\right|_{R\{w\}}$ and therefore

$$
\delta_{\mathrm{D}}^{G}(\sigma)=\left\{\begin{array}{cl}
{[G: H] \cdot \delta_{\mathrm{E}}^{H}(\sigma),} & \sigma \in H \backslash\{1\} \\
0, & \sigma \in G \backslash H .
\end{array}\right.
$$

Now the first equation in (3) is obvious.

Fix an element $\sigma \in G \backslash\{1\}$ and set $f_{\sigma}:=\sigma(z)-z \in R[[z]] \subset R\{w\}$. By the assumption on $\mathrm{D}$, the function $f_{\sigma}$ has no zero on the annulus $\mathrm{Y} \backslash \mathrm{D}$. It follows that

$$
\operatorname{val}_{\mathrm{D}}\left(f_{\sigma}\right)=\operatorname{val}_{\mathrm{Y}}\left(f_{\sigma}\right)+\epsilon \cdot \#_{\mathrm{Y}} f_{\sigma},
$$

see e.g. [1], §4.5. We compute:

$$
\begin{aligned}
\delta_{\mathrm{D}}^{G}(\sigma) & =-|G| \cdot \operatorname{val}_{\mathrm{D}}(\sigma(w)-w)=-|G| \cdot\left(\operatorname{val}_{\mathrm{D}}\left(f_{\sigma}\right)-\epsilon\right) \\
& \stackrel{(4)}{=}-|G| \cdot \operatorname{val}_{\mathrm{Y}}\left(f_{\sigma}\right)-|G| \cdot \epsilon \cdot\left(\# \mathrm{Y}\left(f_{\sigma}\right)-1\right) \\
& =\delta_{\mathrm{Y}}^{G}(\sigma)+|G| \cdot \epsilon \cdot s_{\mathrm{Y}}^{G} .
\end{aligned}
$$


This proves the second equation in (3).

3.4 Definition of the Hurwitz tree We can now state and prove our main theorem.

Theorem 3.7 Let $\mathrm{Y}=(\operatorname{Spf} R[[z]]) \otimes K$ be an open rigid disk over $K$ and $G \subset \operatorname{Aut}_{K}(\mathrm{Y})$ be a finite group of automorphisms. Suppose that the set of fixed points $\Delta \subset \mathrm{Y}$ is nonempty. Then there exists a Hurwitz tree $\mathcal{T}$ of type $(G, p)$ with

$$
\delta_{\mathcal{T}}=\delta_{Y}^{G}, \quad a_{\mathcal{T}}=a_{Y}^{G} .
$$

Proof: Our proof is by induction over the number of elements of $\Delta$.

We first assume that $|\Delta|=1$. In this case the theorem is essentially equivalent to the following lemma.

Lemma 3.8 Let $y \in \Delta$ be the unique fixed point. Then

(i) the group $G$ is cyclic,

(ii) $a_{Y}^{G}=u_{G}$, and

(iii) $\delta_{Y}^{G}=\left(\delta_{P}^{\text {mult }}\right)^{*}$, where $P$ is the Sylow $p$-subgroup of $G$.

Proof: It is clear that every element of $G$ fixes the point $y$. So (ii) follows directly from Proposition 3.5.

After a change of parameter we may assume that $y$ is the point $z=0$. Then for an element $\sigma \in G$ we have

$$
\sigma(z)=\chi(\sigma) z\left(1+a_{1} z+a_{2} z^{2}+\ldots\right)
$$

where $\chi: G \hookrightarrow K^{\times}$is an injective character ([7], §II.1). This proves (i). Let us fix an element $\sigma \in G$ of order $n p^{m}$, with $(n, p)=1$ and $m \geq 0$. By (6) we have

$$
f_{\sigma}:=\sigma(z)-z=(\chi(\sigma)-1) z+\chi(\sigma) a_{1} z^{2}+\ldots
$$

Since $z=0$ is the only zero of $f_{\sigma}$, we have $\# f_{\sigma}=1$ and therefore

$$
\operatorname{val}_{Y}\left(f_{\sigma}\right)=\operatorname{val}(\chi(\sigma)-1)= \begin{cases}0, & m=0 \\ \frac{1}{(p-1) p^{m-1}}, & m>0\end{cases}
$$

Now (iii) follows from Definition 2.1 and a direct computation.

So in the case $|\Delta|=1$ we define the Hurwitz tree $\mathcal{T}=\left(T,\left[G_{v}\right], \delta_{v}, a_{e}\right)$ as follows.

- The tree $T$ has two vertices $v_{0}, v_{1}$ and one edge $e_{0}$ with $s\left(e_{0}\right)=v_{0}$ and $t\left(e_{0}\right)=v_{1}$. The metric $\epsilon$ is trivial, i.e. we set $\epsilon_{e_{0}}:=0$.

- We define

$$
\delta_{v_{0}}=\delta_{v_{1}}:=\delta_{Y}^{G}
$$


- We define $G_{v_{0}}=G_{v_{1}}:=G$ and $a_{e_{0}}:=u_{G}$.

The validity of the axioms (H2) and (H5) follows from Lemma 3.8; all the other axioms and (5) hold by definition. This finishes the proof of the theorem in the case $|\Delta|=1$.

We may now assume that $|\Delta| \geq 2$. Then there exists a smallest closed disk $\mathrm{D} \subset \mathrm{Y}$ which contains $\Delta$. Clearly, $\mathrm{D}$ is fixed by the $G$-action. There also exists a finite family $\left(\mathrm{E}_{j}\right)_{j \in J}$ of residue classes $\mathrm{E}_{j} \subset \mathrm{D}$ with

$$
\Delta_{j}:=\mathrm{E}_{j} \cap \Delta \neq \emptyset \text { and } \Delta \subset \cup_{j} \mathrm{E}_{j} .
$$

For $j \in J$ we let $G_{j} \subset G$ denote the stabilizer of $\mathrm{E}_{j}$. By induction, there exists a Hurwitz tree $\mathcal{T}_{j}$ for the group $G_{j}$ with

$$
\delta_{\mathcal{T}_{j}}=\delta_{\mathrm{E}_{j}}^{G_{j}}, \quad a_{\mathcal{T}_{j}}=a_{\mathrm{E}_{j}}^{G_{j}} .
$$

The Hurwitz tree $\mathcal{T}=\left(T,\left[G_{v}\right], a_{e}, \delta_{v}\right)$ associated to $(\mathrm{Y}, G)$ is defined as follows.

- Let $T_{j}$ denote the metric tree underlying the Hurwitz tree $\mathcal{T}_{j}$. Choose a system of representatives $J^{\prime} \subset J$ of $J / G$. The metric tree $T$ underlying $\mathcal{T}$ is obtained by patching together the metric trees $T_{j}, j \in J^{\prime}$, at their roots, i.e. we identify the set of roots of the trees $T_{j}, j \in J^{\prime}$ with one vertex $v_{1}$ of $T$. We complete $T$ by adding another vertex $v_{0}$ (the root of $T$ ) and an edge $e_{0}$ with $s\left(e_{0}\right)=v_{0}, t\left(e_{0}\right)=v_{1}$. The value of the metric $\epsilon$ on the edge $e_{0}$ is defined as the thickness of the annulus $Y \backslash \mathrm{D}$, multiplied with $|G|$. (In fact, $\epsilon_{e_{0}}$ is the thickness of the quotient annulus $(\mathrm{Y} \backslash \mathrm{D}) / G$.)

- If $v$ is a vertex of $T$ other than $v_{0}$ and $v_{1}$, it corresponds to a vertex $v^{\prime}$ of one of the $T_{j}$ which is not the root. We define $G_{v}:=G_{v^{\prime}}$ and $\delta_{v}:=\delta_{v^{\prime}}^{*}$.

- Let $e$ be an edge of $T$ which corresponds to an edge $e^{\prime}$ of $T_{j}$. We define $a_{e}:=a_{e^{\prime}}^{*}$.

- We set $G_{v_{0}}=G_{v_{1}}:=G, \delta_{v_{0}}:=\delta_{\mathrm{Y}}^{G}, \delta_{v_{1}}:=\delta_{\mathrm{D}}^{G}$ and $a_{e_{0}}:=a_{\mathrm{Y}}^{G}$.

It remains to show that $\mathcal{T}$ satisfies the axioms (H1)-(H5). Since these axioms hold for the Hurwitz trees $\mathcal{T}_{j}$, many of them hold for $\mathcal{T}$ by construction. For instance, this is clear for (H1) and (H2).

It follows from (7), (8) and Proposition 3.5 that

$$
a_{e_{0}}=a_{\Upsilon}^{G}=\sum_{j \in J / G}\left(a_{\mathrm{E}_{j}}^{G_{j}}\right)^{*}=\sum_{s(e)=v_{1}} a_{e} .
$$

Therefore, (H3) holds for the edge $e_{0}$. For the other edges it holds by construction.

To check the axioms (H4) and (H5) we remark that

$$
\delta_{v_{1}}=\delta_{\mathrm{D}}^{G}=\delta_{\mathcal{T}_{j}}^{*}
$$


for all $j \in J$, by the first equality in (3). This means that our definition of $\delta_{v_{1}}$ is consistent with the fact that the vertex $v_{1}$ corresponds to the roots of the Hurwitz trees $\mathcal{T}_{j}, j \in J^{\prime}$. It follows that (H5) holds automatically and that we have to check (H4) only for the edge $e_{0}$. But for the edge $e_{0}$ the statement of (H4) follows directly from Proposition 3.6. This concludes the proof of Theorem 3.7 .

Remark 3.9 An alternative way to construct the metric tree $T$ is the following (cf. [8] and [3]). Let $\mathcal{Y}$ be the minimal semistable model of the disk $Y$ which separates the points of $\Delta$. Then the $G$-action on $Y$ extends to $\mathcal{Y}$, and the quotient $\mathcal{X}:=\mathcal{Y} / G$ is a semistable model of the disk $\mathrm{X}=\mathrm{Y} / G$ which separates the points of $B:=\Delta / G$. Now there is a standard way to associate to the pair $(\mathcal{X}, B)$ a metric tree $T$ with set of leaves $B$ (see e.g. [3], 33.2 ). Essentially, $T$ is a modification of the graph of components of the special fiber of $\mathcal{X}$.

The construction of $T$ in the proof of Theorem 3.7 avoids the use of semistable models and may therefore be considered as more elementary. However, semistable models become inevitable if one wants to construct $G$-actions on the disk with given Hurwitz tree.

\section{Applications to the lifting problem}

4.1 A new obstruction Let $k$ be an algebraically closed field of characteristic $p>0$ and $G$ be a finite group. A local $G$-action is a faithful and $k$-linear action $\phi: G \hookrightarrow \operatorname{Aut}_{k}(k[[z]])$ on a ring of formal power series in one variable over $k$.

The local lifting problem asks: can $\phi$ be lifted to an action $\phi_{R}: G \hookrightarrow$ $\operatorname{Aut}_{R}(R[[z]])$, where $R$ is some discrete valuation ring of characteristic zero with residue field $k$. If it does then we say that $\phi$ lifts to characteristic zero.

From our main result we can deduce a new necessary condition for liftability of local $G$-actions. Before we state it, we recall the definition of the Artin character.

Definition 4.1 Let $\phi$ be a local $G$-action. The Artin character of $\phi$ is the element $a_{\phi} \in R^{+}(G)$ defined by

$$
a_{\phi}(\sigma):=-\operatorname{ord}_{z}(\sigma(z)-z)
$$

for $\sigma \neq 1$ and

$$
a_{\phi}(1):=-\sum_{\sigma \neq 1} a_{\phi}(\sigma) .
$$

See [14], VI, §2.

Theorem 4.2 Let $\phi: G \hookrightarrow \operatorname{Aut}_{k}(k((t)))$ be a local $G$-action. If $\phi$ lifts to characteristic 0 then there exists a Hurwitz tree $\mathcal{T}$ of type $(G, p)$ such that

$$
a_{\mathcal{T}}=a_{\phi} \quad \text { and } \quad \delta_{\mathcal{T}}=0 .
$$


Proof: A lift of $\phi$ gives rise to a $G$-action on the disk $\mathrm{Y}=(\operatorname{Spf} R[[z]]) \otimes K$. Since $\phi$ is injective by assumption, we have $\delta_{Y}^{G}=0$ (Definition 3.1) and $a_{Y}^{G}=a_{\phi}$ (Definition 3.4). Therefore, Theorem 4.2 is a direct consequence of Theorem 3.7 .

By the theorem, the existence of a Hurwitz tree $\mathcal{T}$ with given Artin character $a_{\mathcal{T}}=a_{\phi}$ and trivial depth $\delta_{\mathcal{T}}=0$ is a necessary condition for $\phi$ to lift. If one can show that such a Hurwitz tree does not exist, one has found an obstruction against liftability of $\phi$.

As a special case of this criterion, we obtain the well-known Bertin obstruction, see [2]. Namely, if $\mathcal{T}=\left(T,\left[G_{v}\right], a_{e}, \delta_{v}\right)$ is a Hurwitz tree with $a_{\mathcal{T}}=a_{\phi}$, then Remark 2.5 shows that

$$
a_{\phi}=\sum_{b \in B} u_{G_{b}}^{*} .
$$

This equality is easily seen to imply the following statement: there exists a finite $G$-set $\Delta$, with cyclic stabilizers, such that

$$
a_{\phi}=m \cdot r_{G}-\chi_{\Delta} .
$$

Here $\chi_{\Delta} \in R^{+}(G)$ is the character of the permutation representation realized by $\Delta$ and $m:=|\Delta / G|$. However, there exist local $G$-actions $\phi$ whose Artin character can not be written in this form (see e.g. [2] and [5]). It follows from Theorem 4.2 that such a $\phi$ does not lift to characteristic zero.

Remark 4.3 The examples presented in Section 4.2 show that our new obstruction is strictly stronger than the Bertin obstruction. However, it should be pointed out that the converse of Theorem 4.2 does not hold. For $G=\mathbb{Z} / p \times \mathbb{Z} / p$, Pagot has shown in [11] that certain local $G$-actions $\phi$ do not lift to characteristic zero. For such a $\phi$ it is straightforward to write down a Hurwitz tree $\mathcal{T}$ with $a_{\mathcal{T}}=a_{\phi}$ and $\delta_{\mathcal{T}}=0$.

Remark 4.4 It will be shown in [4] show that our new obstruction vanishes for all cyclic groups $G$, in accordance with Oort's conjecture (see [5]). The interesting thing about the proof is that one has to use certain nontrivial inequalities satisfied by the Artin character of a local action of a cyclic group of order $p^{n}$ (see [12]). We believe that this observation provides further substantial evidence in favour of Oort's conjecture.

4.2 Simple quaternion actions We fix an integer $n \geq 2$ and let $G=Q_{2^{n+1}}$ denote the generalized quaternion group of order $2^{n+1}$, with presentation

$$
G=\left\langle\sigma, \tau \mid \tau^{2^{n}}=1, \tau^{2^{n-1}}=\sigma^{2}, \sigma \tau \sigma^{-1}=\tau^{-1}\right\rangle .
$$

Our base field $k$ is assumed to be of characteristic 2 .

Chinburg, Guralnick and Harbater ([5]) have proved that $G$ is a local Bertin group for $n \geq 3$, which means that the Bertin obstruction of every local $G$ action over $k$ vanishes. The goal of this section is to construct certain $G$-actions 
which do not lift to characteristic zero. This result gives a negative answer to Question 1.3 of [5].

We first introduce some more notation. Set

$$
H_{0}:=\langle\tau\rangle, \quad H_{1}:=\langle\sigma\rangle, \quad H_{2}:=\langle\sigma \tau\rangle ;
$$

these are cyclic subgroups of $G$ of order $2^{n}, 4$ and 4 , respectively. For $i=0,1,2$ there exists a unique character $\chi_{i}: G \rightarrow\{ \pm 1\}$ of order 2 such that $H_{i} \subset \operatorname{Ker}\left(\chi_{i}\right)$. Clearly, $\chi_{0}, \chi_{1}, \chi_{2}$ define pairwise distinct irreducible characters of the quotient group

$$
\bar{G}:=G /\left\langle\tau^{2}\right\rangle \cong \mathbb{Z} / 2 \times \mathbb{Z} / 2 .
$$

The following lemma is an easy exercise:

Lemma 4.5 If $H \subset G$ is a cyclic subgroup whose image in $\bar{G}$ is nontrivial, then $H$ is conjugate to one of the groups in (13).

Definition 4.6 A local $G$-action $\phi$ is called simple if

$$
a_{\phi}\left(\chi_{0}\right)=2, \quad a_{\phi}\left(\chi_{1}\right)=a_{\phi}\left(\chi_{2}\right) \geq 2 .
$$

Proposition 4.7 There exists a simple $G$-action over $k$, for every $n \geq 2$.

Proof: Choose an embedding of abelian groups $\bar{G} \hookrightarrow(k,+)$. We obtain a local $\bar{G}$-action $\bar{\phi}: \bar{G} \hookrightarrow \operatorname{Aut}_{k}(k[[t]])$ by sending $\mu \in \bar{G}$ to the automorphism

$$
t \mapsto \frac{t}{1+\mu t}=t-\mu t^{2}+\mu^{2} t^{3}-\ldots
$$

One checks that

$$
a_{\bar{\phi}}\left(\chi_{i}\right)=2, \quad \text { for } i=0,1,2 .
$$

By [6], Lemma 2.10, we can extend $\bar{\phi}$ to a local $G$-action $\phi: G \hookrightarrow \operatorname{Aut}_{k}(k[[z]])$, such that $k[[t]]=k[[z]]^{\left\langle\tau^{2}\right\rangle}$. It follows from [14], Proposition IV.3, that

$$
a_{\phi}\left(\chi_{i}\right)=a_{\bar{\phi}}\left(\chi_{i}\right), \quad i=0,1,2 .
$$

We conclude that $\phi$ is simple.

Theorem 4.8 Let $\phi$ be a simple $G$-action over $k$. Then $\phi$ does not lift to characteristic zero.

Proof: Suppose that $\phi$ lifts to characteristic zero. By Theorem 4.2, there exists a Hurwitz tree $\mathcal{T}=\left(T,\left[G_{v}\right], a_{e}, \delta_{v}\right)$ of type $(G, 2)$, with Artin character $a_{\mathcal{T}}=a_{\phi}$ and vanishing depth $\delta_{\mathcal{T}}=0$. We will show that such a Hurwitz tree cannot exist. Our main tool is the notion of density introduced in Section 2.4.

Let $B$ denote the set of ends of the tree $T$. For $i=0,1,2$ we set

$$
B_{i}:=\left\{b \in B \mid\left[G_{b}\right]=\left[H_{i}\right]\right\}, \quad B^{\prime}:=B_{0} \dot{\cup} B_{1} \dot{\cup} B_{2}
$$


and

$$
B^{i}:=B^{\prime} \backslash B_{i} .
$$

It follows from Lemma 4.5 and the definition of the groups $H_{i}$ that

$$
\left\langle\chi_{i}, u_{G_{b}}^{*}\right\rangle= \begin{cases}1, & b \in B^{i} \\ 0, & b \in B \backslash B^{i},\end{cases}
$$

for all $b \in B$. So Lemma 2.7 (ii), Condition (H5) of Definition 2.4 and (1) show that

$$
d\left(B^{i}, b\right)=\delta_{b}\left(\chi_{i}\right)=2,
$$

for all $b \in B^{i}$.

From (11) and (14) we conclude that

$$
a_{\phi}\left(\chi_{i}\right)=\left|B^{i}\right|=\sum_{j \neq i}\left|B_{j}\right| .
$$

Now the assumption that $\phi$ is simple (Definition 4.6) implies that

$$
\left|B_{1}\right|=\left|B_{2}\right|=1, \quad\left|B_{0}\right| \geq 1 .
$$

Let $b_{0}$ denote the unique element of $B_{2}$. Since the $B_{i}$ are disjoint, we have $B^{0} \cap B^{1}=B_{2}=\left\{b_{0}\right\}$ and $B^{0} \cup B^{1}=B^{\prime}$. Using Definition 2.6 (ii) and (15) we therefore get

$$
d\left(B^{\prime}, b_{0}\right)=d\left(B^{0}, b_{0}\right)+d\left(B^{1}, b_{0}\right)=2+2=4 .
$$

Let $\chi: H_{0} \hookrightarrow \mathbb{C}^{\times}$be an injective irreducible character. The induced character $\psi:=\chi^{*} \in R^{+}(G)$ has the following property. For any nontrivial cyclic subgroup $C \subset G$, the restriction $\left.\psi\right|_{C}$ is the sum of two nontrivial irreducible characters of $C,\left.\psi\right|_{C}=\psi_{1}+\psi_{2}$. Applying this to $C=G_{b}$, we obtain

$$
\left\langle\psi, u_{G_{b}}^{*}\right\rangle_{G}=\left\langle\psi_{1}, u_{G_{b}}\right\rangle+\left\langle\psi_{2}, u_{G_{b}}\right\rangle=2,
$$

for all $b \in B$. We may therefore apply Lemma 2.7 (ii) and conclude that

$$
d\left(B, b_{0}\right)=\delta_{b_{0}}(\psi) / 2 .
$$

Moreover, the restriction of $\psi$ to $G_{b_{0}}=H_{2} \cong \mathbb{Z} / 4$ is the sum of two irreducible characters $\psi_{1}, \psi_{2}$ of order 4 . From (H5) and (1) we get

$$
\delta_{b_{0}}(\psi)=\delta_{H_{2}}^{\text {mult }}\left(\psi_{1}\right)+\delta_{H_{2}}^{\text {mult }}\left(\psi_{2}\right)=3+3=6 .
$$

We now obtain a contradiction by comparing (17), (18) and (19):

$$
4=d\left(B^{\prime}, b_{0}\right) \leq d\left(B, b_{0}\right)=3 .
$$

We conclude that there does not exist a Hurwitz tree $\mathcal{T}$ of type $(G, 2)$ with $a_{\mathcal{T}}=a_{\phi}$ and $\delta_{\mathcal{T}}=0$. Theorem 4.8 follows. 


\section{References}

[1] Y. Amice. Les nombres p-adiques. Presses Univ. France, 1975.

[2] J. Bertin. Obstructions locales au relèvement de revêtements galoisiens de courbes lisses. C.R. Acad. Sci. Paris, Série I, 326:55-58, 1998.

[3] I. I. Bouw and S. Wewers. The local lifting problem for dihedral groups. Duke Math. J., 134:421-452, 2005.

[4] L.H. Brewis. Swan characters, Hurwitz trees and the local lifting problem. PhD thesis, Ulm University, 2009 (expected).

[5] T. Chinburg, R. Guralnick, and D. Harbater. The local lifting problem for actions of finite groups on curves. Preprint, available at www . math. upenn. edu/ ted/manuscripts/locallift.pdf.

[6] T. Chinburg, R. Guralnick, and D. Harbater. Oort groups and lifting problems. Compositio Math., 144(4):849-866, 2008.

[7] B. Green and M. Matignon. Order $p$ automorphisms on the open disc of a p-adic field. J. Amer. Math. Soc., 12:269-303, 1999.

[8] Y. Henrio. Arbres de Hurwitz et automorphismes d'ordres $p$ des disques et des couronnes $p$-adiques formelles. arXiv:math. AG/0011098.

[9] Y. Henrio. Automorphismes d'ordre $p$ des couronnes $p$-adiques ouvertes. C. R. Acad. Sci. Paris Sér. I Math., 329(1):47-50, 1999.

[10] R. Huber. Swan representations associated with rigid analytic curves. J. Reine Angew. Math., 537:165-234, 2001.

[11] G. Pagot. $\mathbb{F}_{p}$-espaces vectoriels de formes différentielles logarithmiques sur la droite projective. J. Number Theory, 97(1):58-94, 2002.

[12] H.L. Schmidt. Zur Arithmetik der zyklischen p-Körper. J. reine angew. Math., 176:161-167, 1937.

[13] J.-P. Serre. Representations Linéaires des Groupes Finis. Hermann, 1967.

[14] J.-P. Serre. Corps Locaux. Hermann, 1968.

Institut für Reine Mathematik

Universität Ulm

Helmholtzstr. 18

89069 Ulm

louis.brewis@uni-ulm.de
IAZD

Leibniz-Universität Hannover

Welfengarten 1

30167 Hannover

wewers@math.uni-hannover.de 